\title{
Maxillary Sinus Augmentation by Autogenous Block Graft A- Case Report
}

\author{
Dr. Tazeen Dawood ${ }^{1}$, Dr. Hassan Sadek ${ }^{2}$. \\ ${ }^{I}$ Assistant Professor, Department Of Periodontics, College Of Dentistry, Jazan University, \\ Jazan, Saudi Arabia. \\ ${ }^{2}$ Professor, Department Of Oral And Maxillofacial Surgery And Implantology, College of Dentistry, \\ Jazan University, Jazan, Saudi Arabia.
}

\begin{abstract}
Tooth morbidity and tooth mortality are one of the most prevalent disease condition worldwide. When a tooth is lost, whether through dental decay, periodontal disease or dental trauma, the alveolar process begins to remodel. Continuous bone remodeling, absence of stimulation, loss of bone height, and density leads to an increase in antral pneumatization, leading to reduction in residual vertical bone height thus making standard implant placement difficult. To adapt, circumvent, and treat this limitation; maxillary sinus floor elevation is often done and has become an important preplacement procedure in dental implant treatment planning. In this article, we present a unique case of maxillary sinus augmentation of right posterior maxilla using a combination of autogenous chin block graft, and allograft with simultaneous placement of endosseous implants.
\end{abstract}

Keywords: autograft, allograft, maxillary sinus augmentation

\section{Introduction}

Maxillary sinus floor augmentation (also termed sinus lift, sinus graft, sinus augmentation or sinus procedure) is a surgical_procedure which aims to increase the amount of bone in the posterior maxilla including premolar, ${ }^{,}$and molar teeth, the indications of this modality include absence of significant amount of bone in the posterior maxilla, those who have missing teeth due to genetics or birth defect, patients with maxillary vertical bone height $<10 \mathrm{~mm}$, systemically healthy, non diabetic and non smokers with good condition of remaining dentition. $[1,2]$ The contra- indications include presence of tumours, maxillary sinus infections, severe chronic sinusitis, deformity of sinus from previous surgery, severe allergic rhinitis, chronic use of topical steroids and radiation therapy. Maxillary sinus elevation is carried out by two approaches - direct maxillary sinus elevation and indirect maxillary sinus elevation. Few of the techniques involve simple, minimal elevation of schneiderian membrane, while others include placement of various types of grafts. Maxillary sinus floor augmentation procedure was first performed by Oscar_Hilt_Tatum,_Jr. in 1974and was originally used as a preprosthetic surgical procedure for patients with large maxillary tuberosity and pneumatized sinuses, in order to reduce the size of maxillary tuberosity without creating an oral-antral defect, bone was grafted into the sinus cavity[2]. Boyne and James were the first to describe the use maxillary sinus bone grafting procedure[3]. The maxillary sinus can be approached through four different anatomic locations 1. superior lateral wall or Caldwell-Luc opening located just anterior to zygomatic arch 2. the middle lateral wall opening located midway between the alveolar ridge and the zygomatic arch 3. Inferior lateral wall opening located at the level of the alveolar ridge and 4. Crestal approach through osteotomes. Among these the most common approach is through the lateral wall. Indirect sinus elevation is done when the remaining alveolar bone height is between $7-10 \mathrm{~mm}$ by osteotome to compress bone internally from the alveolar crest upward against the floor leading to a inward fracture, with the schnederian membrane being intact, an average increases in bone height would be between 2 to $7 \mathrm{~mm}$.Pre-surgical evaluation of the maxillary sinus primary should be accomplished by radiographs including OPG to evaluate any pathology, masses or presence of septa. The decision of simultaneous implant placement depends upon the quality and quantity of the existing native bone.[3,4]

\section{Case Presentation}

A 40-year-old female systemically healthy patient moderately built and moderately nourished reported to the oral implantology unit of College of dentistry Jazan university ,Jazan, Saudi arabia with a chief complaint of missing maxillary right posterior teeth and desired to replace it through implants . Patient was cooperative and did not have any relevant medical history, the entire treatment plan was explained to the patient and a consent form was signed. Clinical and radiographic examination revealed limited vertical alveolar ridge height in relation to $16,17,18$. Direct maxillary sinus lifting was done by lateral approach with autogeneous symphyseal bone grafting followed by implant placements, suture removal was done after one week, patient was 
recalled for check up at monthly intervals followed by abutment placement and final prosthesis at the end of six months.

\section{Investigations}

Pre-surgical evaluation of the maxillary sinus was primary accomplished by OPG to rule out any pathology, masses or presence of septa and to estimate the residual vertical ridge height and width "Fig1". Blood investigation was also done.

\section{Treatment Procedure}

The surgical procedure was carried out under local anaesthesia (2\% mepivacaine with 1:100.000 epinephrine). Maxillary sinus floor augmentation via a lateral approach was performed according to Boyne \& James(1980) technique.[3] The approach to the anterior wall of the maxillary sinus was made by a crestal incision between 15 and 16 and 17 followed by vertical-releasing incisions and the elevation of a mucoperiostal flap "Fig" 2. A bony window on the lateral wall of right maxillary sinus was established with a small diamond round bur under sterile saline irrigation "Fig" 3, taking care not to tear the Schneiderian's membrane. The antral mucosa was elevated inferiorly, anteriorly and posteriorly together with the bony window until the desired elevation was reached "Fig4,5". Sinus floor augmentation was performed with autogenous chin block graft "Fig 6,7," followed by three simultaneous implant placements, (Vitane implants of size $3.6 \times 10 \mathrm{~mm}$ ), allograft namely cerabone granules of particle size $0.5-1 \mathrm{~mm}$ was used to fill the defect ,collagen membrane cytoplast was placed to cover the lateral window "Fig 8 " Wound closure was done with -non resorbale synthetic 4-0 suture "Fig "9. Donor site was packed with cerabone granules."fig "10.Immediate post operative panorama was taken to check the implant directions "Fig11". Post surgical instructions were given along with 1g augmentin, $600 \mathrm{mg}$ brufen and mouthwash for a week. Sutures were removed after 7 days. Four months after ossteointegration of implants abutments were placed, followed by final prosthesis after 6 months.

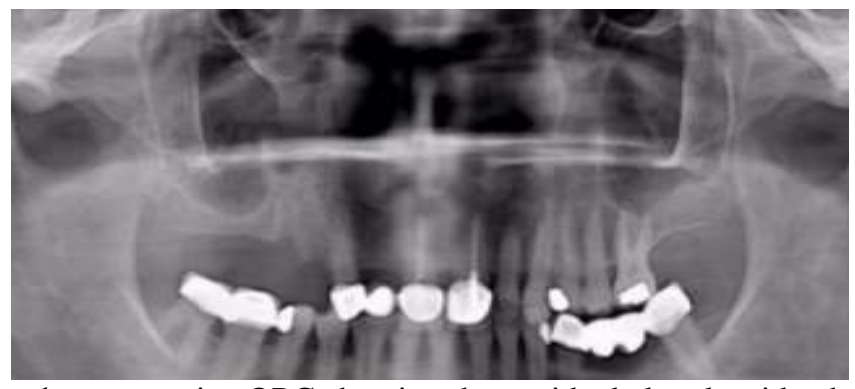

Figure1pre-operative OPG showing the residual alveolar ridge height

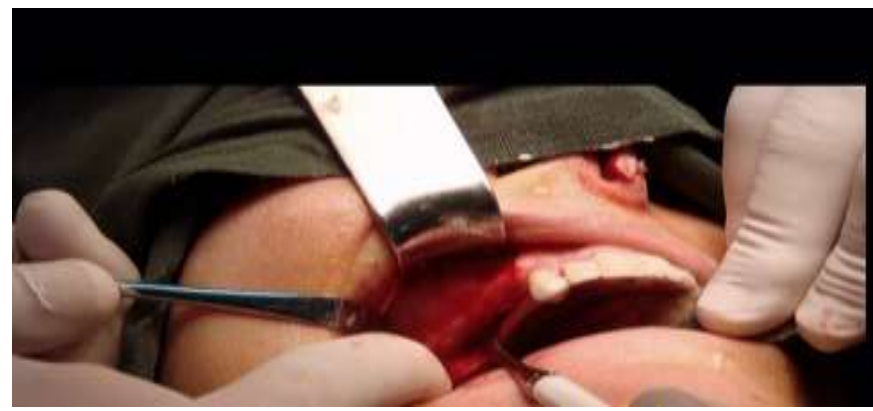

Figure 2 intra oral view showing the residual alveolar ridge after elevation of flap

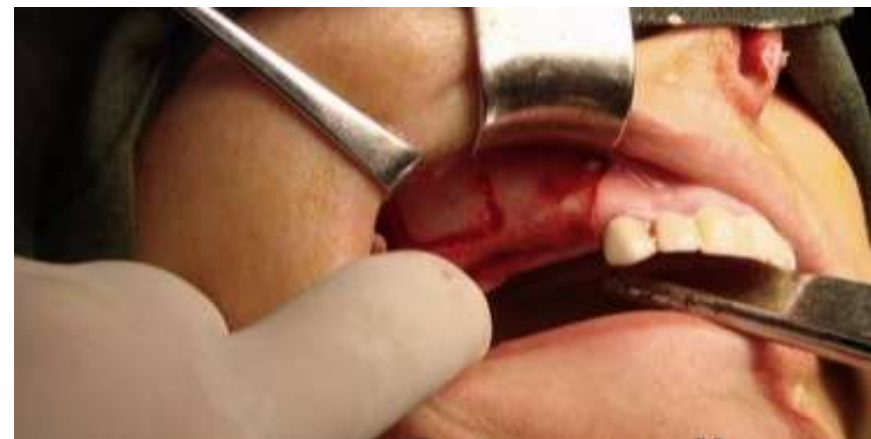

Figure 3 surgical approach to maxillary sinus deplicting the lateral window 


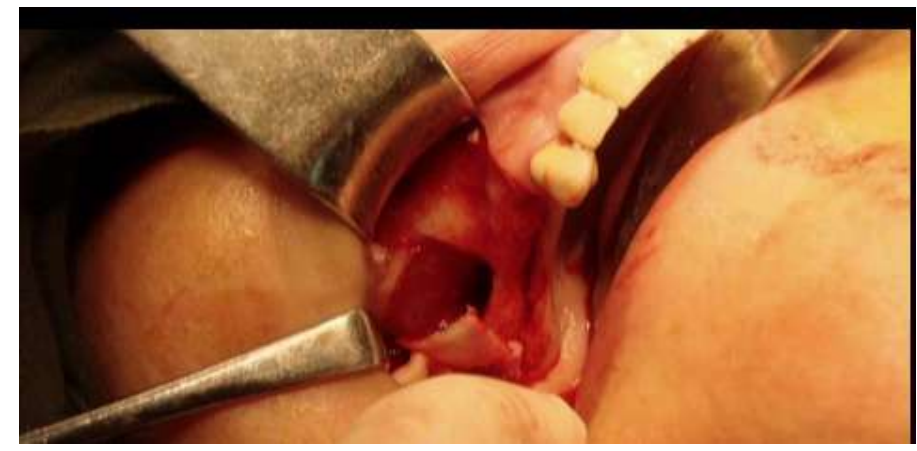

removal of lateral window

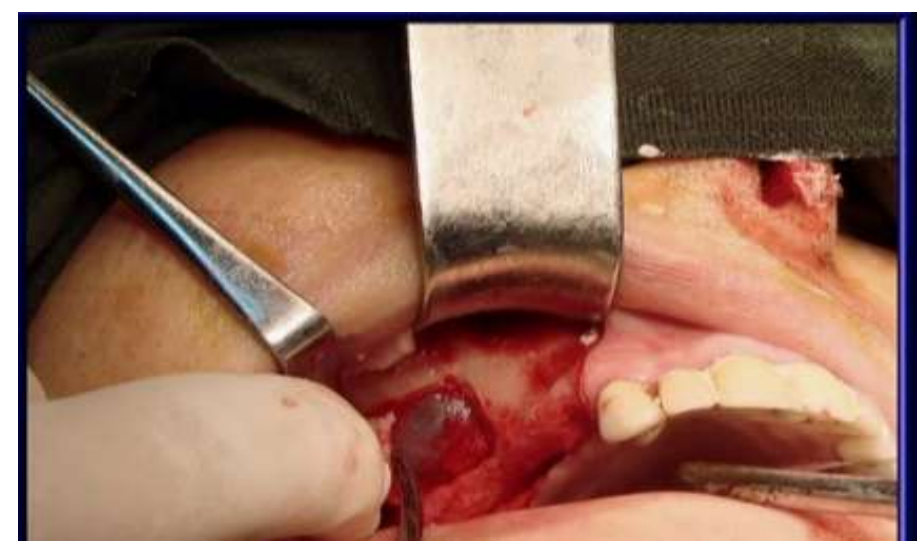

Figure4,5,visible schneiderian membrane

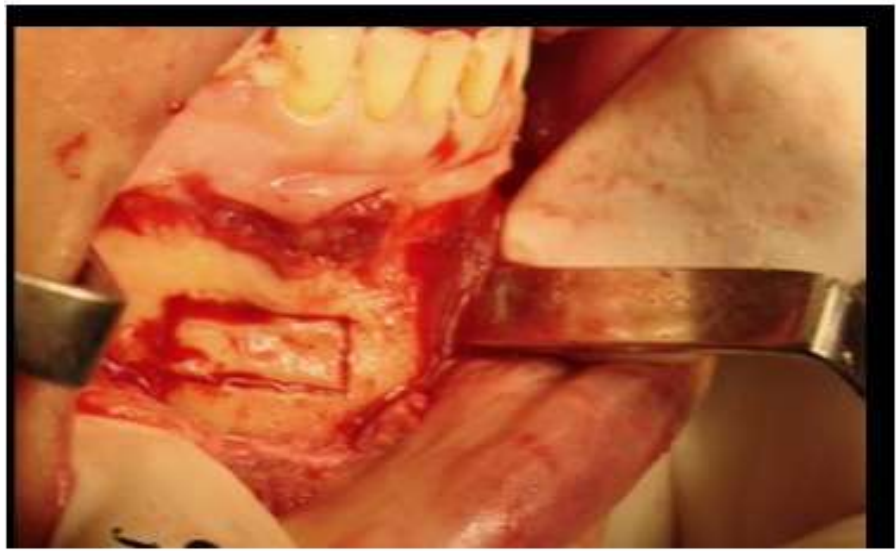

Procuretment of autogenous block graft

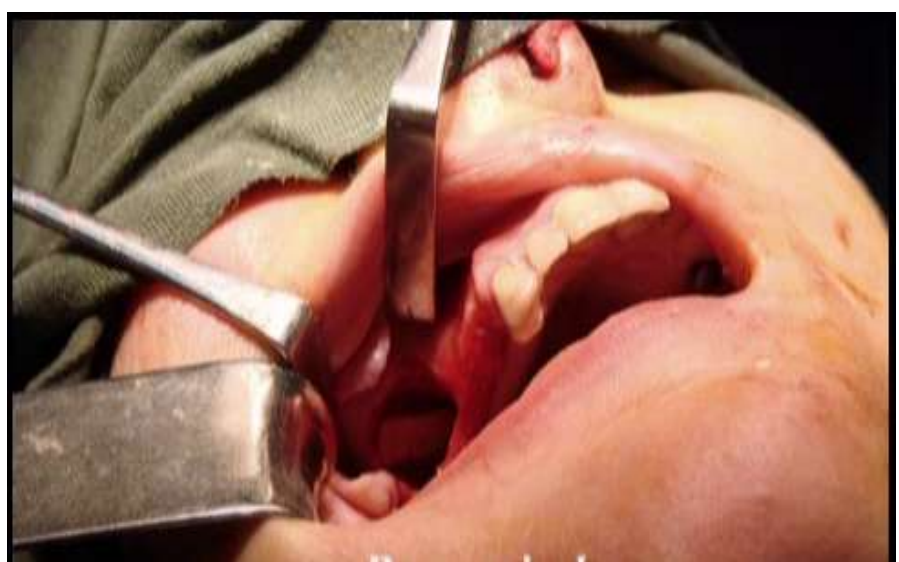

Figure 9 block bone used to elevate the sinus membrane 


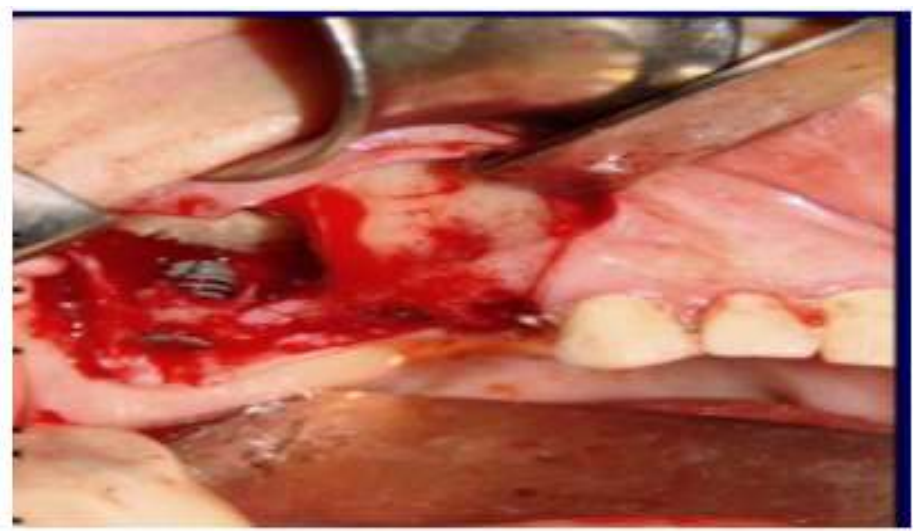

Figure 10 implants placed simultaneously

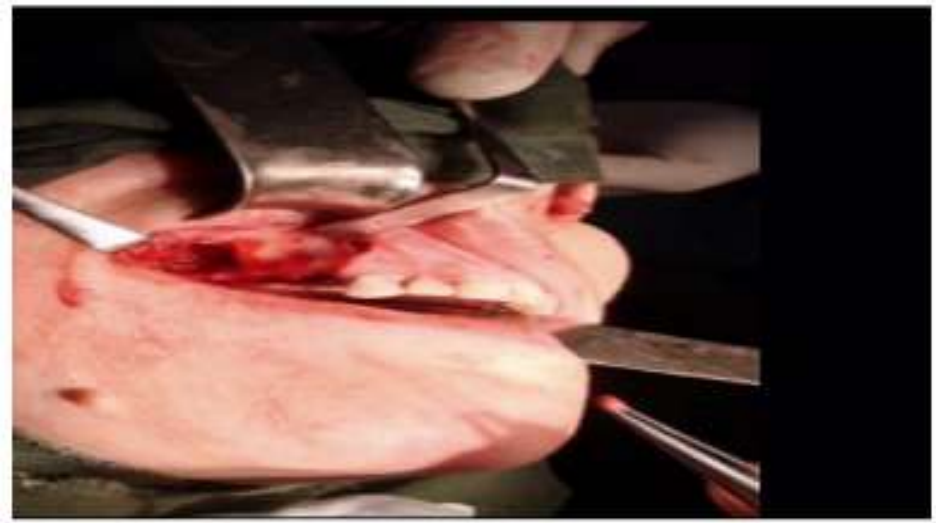

Figure11 allograft cerabone was used to fill the lateral window after implant placement

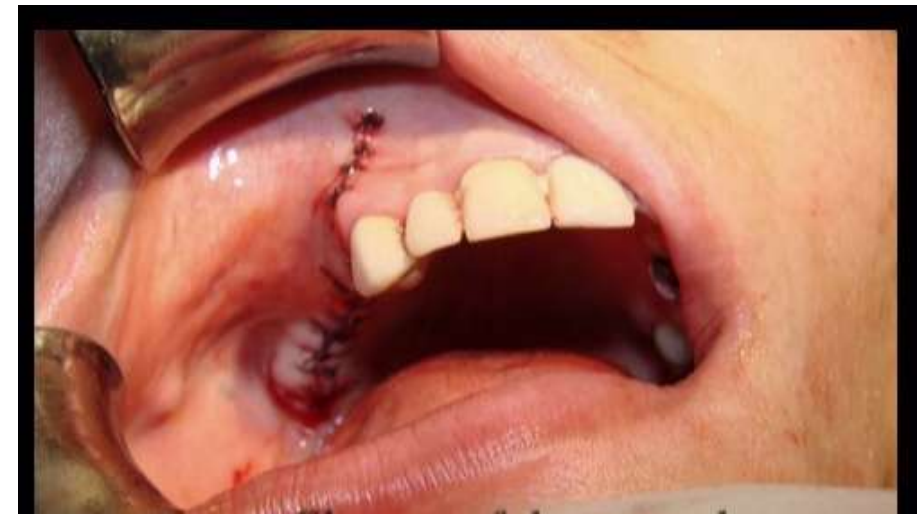

Figure12 flaps approximated and sutures placed

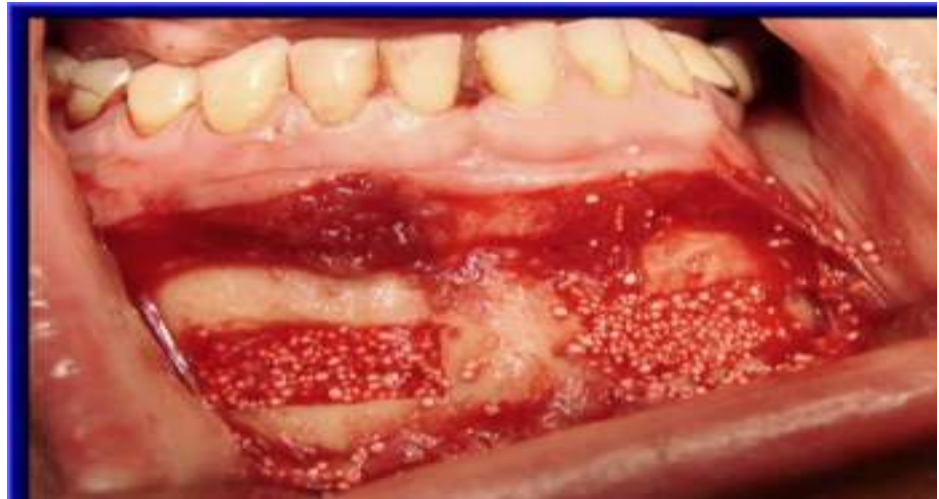

Figure 13 donor site packed with bone allograft- cerabone 


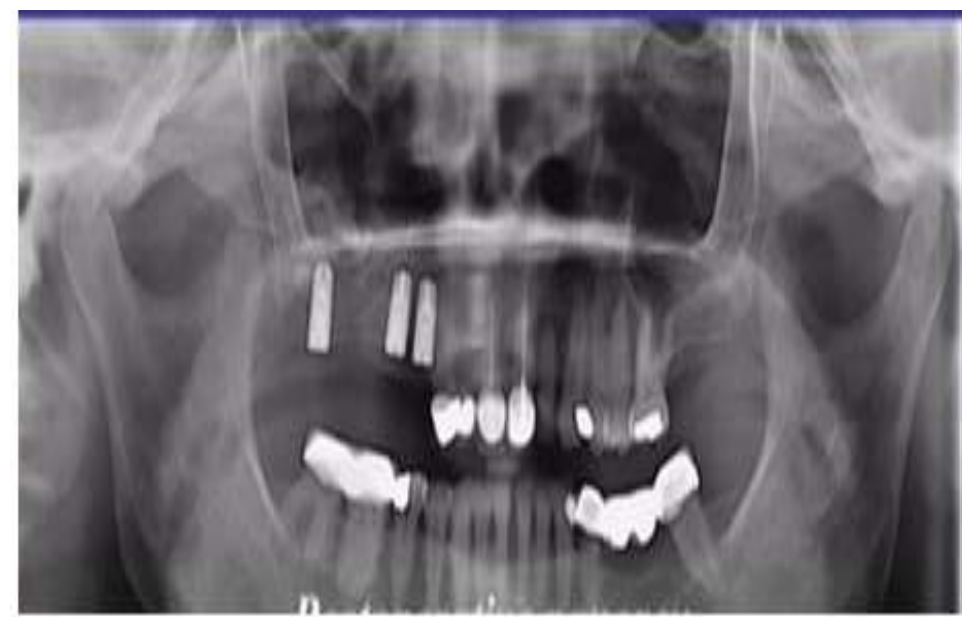

Figure 14 immediate post operative OPG

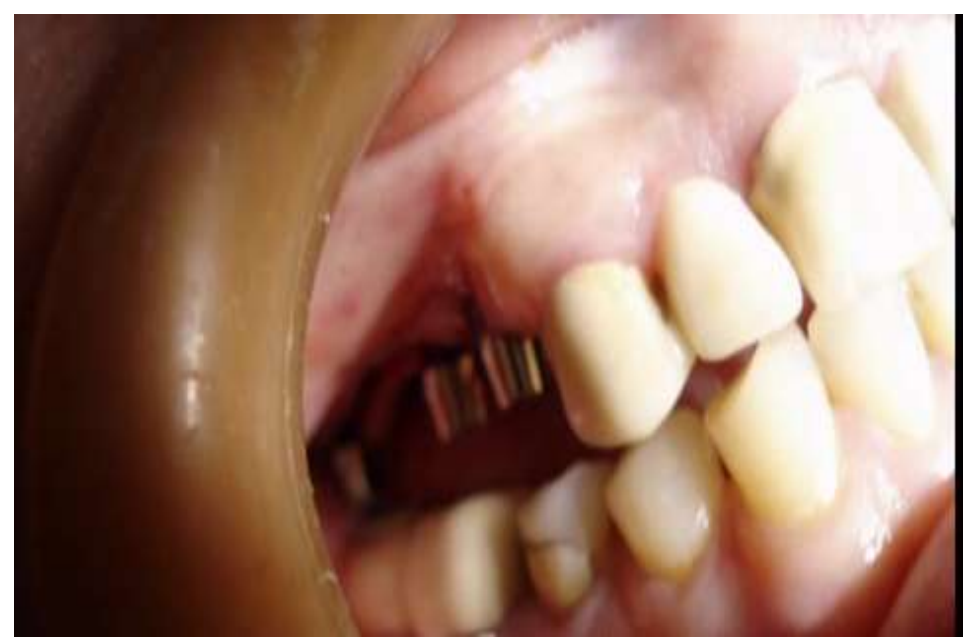

Figure 15 abutments placed after 4 months

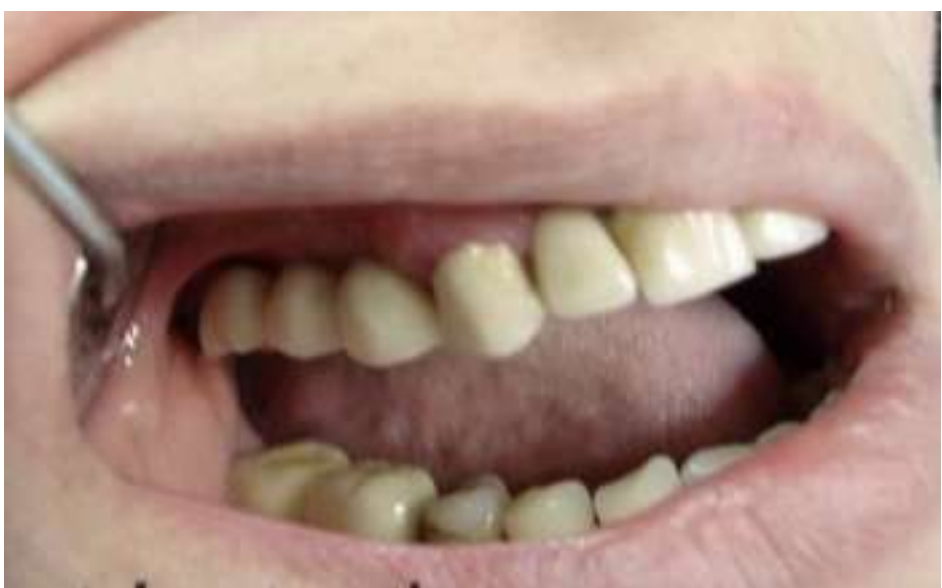

Figure16 final prosthetic procelin crowns placed

\section{Outcome And Follow-Up}

Sinus graft showed full integration during the follow-up period, the patient showed no change in sinus functions. Clinical evaluation showed a normal peri-implant probing depth and no signs of bleeding on probing. Implants were healed with cover screws exposed. The panoramic X-ray after implants placement showed the right implant axis and the stability of the bone graft. After soft-tissue healing, implants were loaded with cemented fixed single crowns supported by pre-formed titanium abutment "Fig"12. The patient was included in a recall program that provides a clinical control every 4 months and radiographic control every 6 months. 


\section{Discussion}

A variety of surgical techniques have been developed to reconstruct the posterior maxilla when bone volume is insufficient. The surgical procedures to obtain bone augmentation are: Guided bone regeneration (GBR); 2) Onlay bone grafts; 3) Inlay grafts; 4) Bone splitting for ridge expansion (RE); 5) Distraction osteogenesis (DO); and 6) Revascularized flaps. Agaloo2008[4], Chiapasso M 2006[5],Vander mark el 2006[6] evaluated the implant success rates of augmentation procedures and survival rates of implants placed in the augmented sites . The implant survival rate was $95.5 \%$ for GBR, $90.4 \%$ for OVG, $94.7 \%$ for DO, and $83.8 \%$ for COG. Vander mark et.al 2011 [7] had conducted a study to compare the implant survival rates of le-fort 1 down grafting and conventional raising of sinus floor with inlay grafting and found no stastistical difference in implant survival rate between the two treatment approaches. In this article, we present a unique case of maxillary sinus lift augmentation of posterior maxilla using autogenous symphyseal block graft with simultaneous implant placement. The use of corticocancellous bone grafts for ridge augmentation in implant dentistry was first reported by Breine and Branemark.[8] Autograft is considered as the gold standard for bone transplantation[9] and various studies have shown efficacy for the same.[10,11,12,13, ].The advantages of corticocancellous block grafts over synthetic grafts are, enhanced revascularization of the cancellous portion, mechanical support and rigidity of the cortical portion, which ensures optimal ridge augmentation.[14,15] The healing of autogenous block grafts has been described as "creeping substitution" where viable bone replaces the necrotic bone within the graft and is highly dependent on graft angiogenesis and revascularization.[17,18]

Several studies have been documented by Kent and Block et.al 1989[19,20], Van Steen Berghe 1997[21] who have conducted retrospective studies on maxillary sinus augmentation using autografts and immediate implant placement which were followed for a period of 2 years and had proved a implant success of $100 \%$.[22] Keestra JA1, et.al 2016 reviewed the different techniques used to augment vertical bone height over a long period of time based on the available data in the current existing studies with a follow-up period of at least 4 to 5 years, they summarized that the onlay technique, alveolar distraction, and vertical guided bone regeneration are stable for at least 4 to 5 years.[23]

\section{Conclusion}

The maxillary sinus augmentation procedure has been well documented. The alveolar ridge augmentation procedures are more technique-sensitive . More in-depth, long-term, multicenter studies are required to provide further insight into augmentation procedures to support dental implant survival. Combination of autogenous graft, and allograft for horizontal and vertical augmentation with simultaneous placement of implants resulted in better functional and esthetic restorations.

\section{References}

[1]. Boyne, PJ. De novo bone induction by recombinant human bone morphogenetic protein-2 (rhBMP-2) in maxillary sinus floor augmentation. J Oral Maxillofac Surg 2005;63:1693-1707

[2]. Tatum H Jr. "Maxillary and sinus implant reconstructions". Dent Clin North Am (1986). 30: 207-229

[3]. Boyne PJ, James RA "Grafting of the maxillary sinus floor with autogenous marrow and bone". J Oral Surg 1980)38: 613-616.

[4]. Aghaloo TL ${ }^{1}$, Moy PK Which hard tissue augmentation techniques are the most successful in furnishing bony support for implant placement?. Int J Oral Maxillofac Implants. 2007;22 Suppl:49-70.

[5]. Chiapasco M, Casentini P, Zaniboni M. Bone augmentation procedures in implant dentistry. Int J Oral Maxillofac Implants. 2009;24(Suppl):237-59.

[6]. Von Arx T, Hardt N, Wallkmamm B. The TIME technique: A new method for localized ridge augmentation prior to placement of dental implants. Int J Oral Maxillofac Implants. 1996;11:387-94

[7]. van der Mark EL ${ }^{1}$, Bierenbroodspot F, Baas EM, de Lange JReconstruction of an atrophic maxilla: comparison of two methods. Br J Oral Maxillofac Surg. 2011 Apr;49(3):198-202

[8]. Breine U, Branemark PI. Reconstruction of alveolar jaw bone: An experimental and clinical study of immediate and preformed autologous bone grafts in combination with osseointegrated implants. Scand J Plast Reconstr Surg. 1980;14:23-48

[9]. McAllister BS, Haghighat K. Bone augmentation techniques. J Periodontol. 2007;78:377-96.

[10]. von Arx T, Hardt N, Wallkmamm B. The TIME technique: A new method for localized ridge augmentation prior to placement of dental implants. Int J Oral Maxillofac Implants. 1996;11:387-94.

[11]. von Arx T, Wallkmamm B, Hardt N. Localized ridge augmentation using a micro titanium mesh: A report on 27 implants followed from 1 to 3 years after functional loading. Clin Oral Implants Res. 1998;9:123-30.

[12]. Proussaefs P, Lozada J, Kleinman A, Rohrer MD, McMillan PJ. The use of titanium mesh in conjunction with autogenous bone graft and inorganic bovine bone material (Bio-oss) for localized alveolar ridge augmentation: A human study. Int J Periodontics Restorative Dent. 2003;23:185-95.

[13]. Roccuzzo M, Ramieri G, Spada MC, Bianchi SD, Berrone S. Vertical alveolar ridge augmentation by means of a titanium mesh and autogenous bone grafts. Clin Oral Implants Res. 2004;15:73-81.

[14]. Proussaefs P, Lozada J. Use of titanium mesh for staged localized alveolar ridge augmentation: Clinical and histologichistomorphometric evaluation. J Oral Implantol. 2006;32:237-47.

[15]. Corinaldesi G, Pieri F, Marchetti C, Finn M, Aldini NN, Giardino R. Histologic and histomorphometric evaluation of alveolar ridge augmentation using bone grafts and titanium micromesh in humans. J Periodontol. 2007;78:1477-484.

[16]. Pieri F, Corinaldesi G, Fini M, Aldini MM, Giardino R, Marchetti C. Alveolar ridge augmentation with titanium mesh and a combination of autogenous bone and anorganic bovine bone: A 2 year prospective study. J Periodontol. 2008;79:2093-103. 
[17]. Corinaldesi G, Pieri F, Sapigni L, Marchetti C. Evaluation of survival and success rates of dental implants placed at the time of or after alveolar ridge augmentation with an autogenous mandibular bone graft and titanium mesh: A 3 to 8 year retrospective study. Int J Oral Maxillofac Implants. 2009;24:1119-124.

[18]. Khamees J, Darwiche MA, Kochaji N. Alveolar ridge augmentation using chin bone graft, bovine bone mineral, and titanium mesh: Clinical, histological, and histomorphometric study. J Ind Soc Periodontol. 2012;16:235-40.

[19]. Rosenberg E, Rose LF. Biologic and clinical considerations for autografts and allografts in periodontal regeneration therapy. Dent Clin North Am. 1998;42:467-90.

[20]. PjeturssonBE,Tan WC,ZwahlenM,LangN.P Asystemic review of the success of sinus floor elevationand survival of implants inserted in combination with sinus floor elevation Clin Periodontol 2008 Sep;35 216-40.

[21]. Kent JN, Block MS. Simultaneous maxillary sinus floor bone grafting and placement of hydroxylapatite-coated implants. J Oral Maxillofac Surg. 1989;47:238-425.

[22]. Van Steenberghe D, Naert I, Bossuyt M, et al.The rehabilitationof the severely resorbed maxilla by simultaneous placementof autogenous bone grafts and implants: A 10-year evaluation.Clin Oral Investig 1997;1:102-108.

[23]. Deshpande $S^{1}$, Deshmukh $\mathrm{J}^{2}$, Deshpande $\mathrm{S}^{3}$, Khatri $\mathrm{R}^{2}$, Deshpande $\mathrm{S}^{4}$. Vertical and horizontal ridge augmentation in anterior

[24]. maxilla using autograft, xenograft and titanium mesh with simultaneous placement of endosseous implants Indian Soc Periodontol. 2014 Sep-Oct; 18(5): 661-665.

[25]. Keestra JA ${ }^{1}$, Barry $\mathrm{O}^{2}$, Jong Ld ${ }^{3}$, Wahl G ${ }^{2}$. J Appl Oral Sci.Long-term effects of vertical bone augmentation: a systematic review.. J Appl Oral Sci. 2016 Jan-Feb;24(1):3-17. 